\title{
Numerical and experimental researches of thermal energy storage processes during phase transformations of phase change materials with nanoparticles
}

\author{
Valery Gorobets ${ }^{1}$, Ievgen Antypov ${ }^{1}$, Yurii Bohdan ${ }^{2, *}$ and Viktor Trokhaniak ${ }^{1}$ \\ ${ }^{1}$ Heat and Power Engineering Department, Education and Research Institute of Energetics, Automation and Energy Efficiency, National \\ University of Life and Environmental Sciences of Ukraine, Heroyiv Oborony st., 15, Kyiv, 03041, Ukraine \\ ${ }^{2}$ Vessel's Power Plant Operation Department, Marine Engineering Faculty, Kherson State Maritime Academy, Ushakova avenue, 20, \\ Kherson, 73000, Ukraine
}

\begin{abstract}
It is evident that nowadays materials with phase or chemical transformations are significantly considered to be in surpassing demand in the society being used for accumulating thermal energy. Furthermore, this usage leads to the process of increasing accumulated thermal energy per unit mass concentration to be achieved in a much beneficial and reliable manner. In comparison to these materials, well-known and widely-used solid and liquid ones are mainly associated with productivity and efficiency losses. One of the methods, having desirable attributes for substantial enhancement in the energy productivity of phase change materials is proved to be the addition of solid nanoparticles with a large coefficient of thermal conductivity.

This work focuses on numerical and experimental investigation the influence of nanoparticles of various materials and sizes on the processes of thermal energy storage in paraffin. In particular, the proceeding data of the thermophysical properties of paraffin with nanoparticles as being found by the optical spectroscopy method. Moreover, due to comprehensive results in experimental and numerical studies, the heat accumulating capacity of the phase change materials, as well as the dynamics and profile of the melting boundary around cylindrical heat sources were reported to be determined. Besides, the comparison of heatstorage capacity for phase change materials with and without nanoparticles is managed.
\end{abstract}

\section{Introduction}

The constant society and industrial growth is conventionally accompanied by prioritizing development needs relating to alternative or renewable energy sources consumption. However, this process faces real challenges caused by the stochastic nature of the work, for example, to take into consideration the phenomenon of uneven absorption of solar energy by solar collectors. Therefore, the ability to control and attempt to stabilize such system operation can be achieved to the full extent both by the exploitation of modern energy-saving equipment and the use of the wide range of accumulators for energy storage purposes. It is worth mentioning that the ongoing variability of heat accumulation method analyses is being introduced by different authors [1-9]. The fundamental approach emphasizes the dominant tendency of heat accumulator consumption of periodic action, which is based on phase or chemical transformations of the accumulation material, as being potentially the most profitable and extensively utilized one.

Indeed, these accumulator types are noticed to be discrepant by the high density of accumulated energy per unit of mass and a stable temperature of the coolant at the outlet of heat accumulator. To be more precise, the detailed information reflecting the processes of heat and mass transfer occurring during phase transformations of phase change material is visually presented in [5-14]. So, according to complex analysis of the conducted research, it can be clearly seen that for the heat accumulator efficiency increasing goals to be attained there is an irreconcilable necessity of having the intensification processes of heat and mass transfer with the "charge" and "discharge" of these accumulators on one hand and the other hand, providing the heat losses reducing while the heat energy is being stored $[5,8,11,12]$. It must be significantly noticed that among all contemporary tendencies, aiming to enhance the heat accumulators built on the principle of phase transformation, one of the superior issues is considered to be the improvement taking into account heat-storage material properties.

Besides, regarding the prevailing attention to the nanotechnologies and their fast development, we certainly emphasize the significance of the ideas of producing new materials and technologies with using of particles of micro- and nano size as being a vital part of studying processes of heat energy accumulation in phase change materials in the presence of nanoparticles in them [15-20].

\footnotetext{
* Corresponding author: bohdanyurii09@gmail.com
} 
However, the contemporary researches relating the questions of the thermophysical properties, concentration, type and size of nanoparticles in the composite heat-storage material have not been extensively investigated. Based on the foregoing, there is clearly a need to carrying out numerical and experimental orientated studies of heat accumulation processes in materials with phase transformations having fillers in the form of nanoparticles with a large coefficient of thermal conductivity. In the experimental research course, a beneficial agreement between the use of traditional methods and current ones is desired to be achieved. For example, modern methods of optical spectroscopy are assumed to be appropriate in this way as they provide the great possibility to study the processes of structural phase transformations in homogeneous and composite materials and to determine the number of important characteristics of these materials [21].

\section{Methodology}

Investigation of the influence of micro- and nanoparticles of metal materials on the intensity of processes of structural-phase transformations of pure and composite paraffin carried out on a developed experimental installation (Figure 1). As it is clearly seen, it consists of two identical experimental modules, one of which is to be filled with pure phase transition material (T3 paraffin) and the second one is to be imbued with the same material and nanoparticles with a high coefficient of thermal conductivity. As the example of that material, micro- and nanoparticles of metal (copper) are proposed to be used. It must be italicized that the plant is additionally equipped with a cylindrical heat energy source (HES) having a power of $1.5 \mathrm{~kW}$, a laboratory autotransformer, a gauging set and equipment for temperature measuring purposes using thermocouples placed on the surface of the HES and in the volume of heat-accumulating material (HAM). As for the experimental modules, they are represented by horizontally oriented capacities, having the case made in the form of a parallelepiped. It was constructed with the precise tolerance to the specified dimensions according to the following parameters: height $\mathrm{H}=120 \mathrm{~mm}$, length $\mathrm{L}=162 \mathrm{~mm}$, width $\mathrm{B}=112 \mathrm{~mm}$.

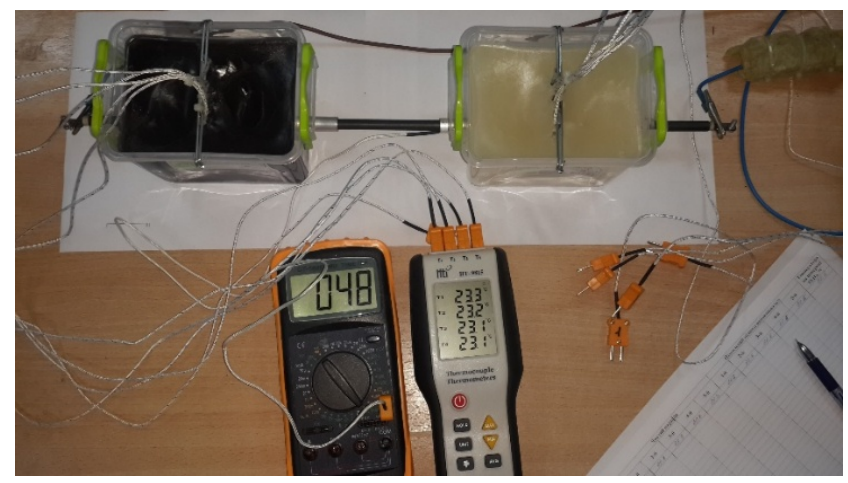

Fig. 1. General view of the experimental installation.
The mass of the accumulative material (paraffin) in the working chamber is adopted to be equal to $\mathrm{M}_{\mathrm{hm}}=$ $749.9 \mathrm{~g}$. Besides, for the measuring temperature procedures, carried out on the surface of a cylindrical heat source, the K-type thermocouples are used. This abovementioned type of heat source, being located in the middle of the volume at a distance of $27 \mathrm{~mm}$ from the bottom of the case and in the volume of the accumulative material, has a diameter of $12 \mathrm{~mm}$. Furthermore, in the control process of temperature fields, precisely speaking in the HAM volume, 5-way thermocouples are involved creating the so-called "temperature grid" (Figure 2). This "grid" was performed in a particular form of being a frame with fixed thermocouples on it and having inside positioned modules placed at a distance of $80 \mathrm{~mm}$ from their ends. As a result, definitely, this arrangement provided an opportunity for the dynamics of temperature fields in the experimental modules to be successfully traced.

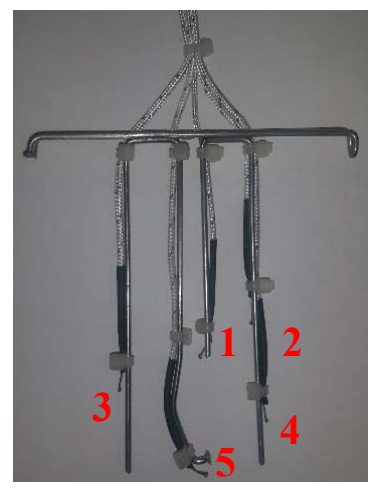

a

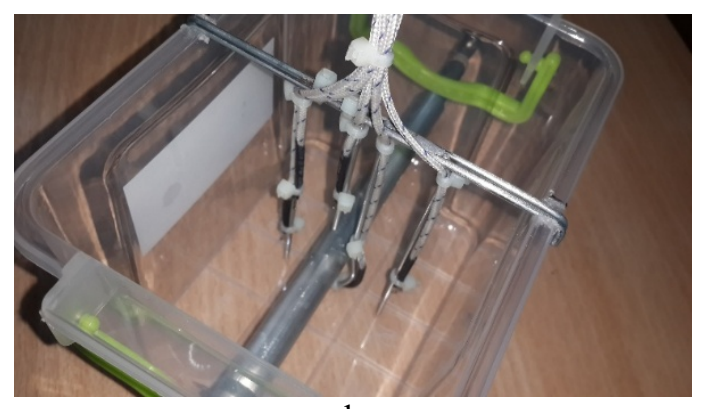

b

Fig. 2. The placement of thermocouples on a

"temperature grid": a - "temperature grid"; b - the location of the "temperature grid" in the experimental module.

The location of thermocouples in the volume of HAM is visually presented in Table 1 .

Table 1. The location of the thermocouples in the HAM $(* "$-" sign indicates that the thermocouples are located under the heat source)

\begin{tabular}{|c|c|c|}
\hline $\begin{array}{c}\text { Thermocouple } \\
\text { Number }\end{array}$ & \multicolumn{2}{|c|}{ Distance, $\times 10^{-2} \mathrm{~m}$, along: } \\
\hline & Horizontal & Vertical \\
\hline 1st & 0.0 & 25.0 \\
\hline 2nd & 15.0 & 15.0 \\
\hline 3rd & 25.0 & 0.0 \\
\hline 4th & $-18.0^{*}$ & $-13.0^{*}$ \\
\hline 5th & 0.0 & $-25.0^{*}$ \\
\hline
\end{tabular}


Therewith, the spectroscopy method of Raman scattering (Raman spectroscopy) can be profitably applied with the aim to define the influence of temperature and impurities on the structural transformations of heat-accumulating substances experimentally. Being a non-destructive method for the reflection of crystallization processes it can analyse thermodynamic properties. The thought is particularly being given to using pure paraffin of brand $\mathrm{T} 3$ as a starting material. The general view of the spectrometer is presented in Figure 3.
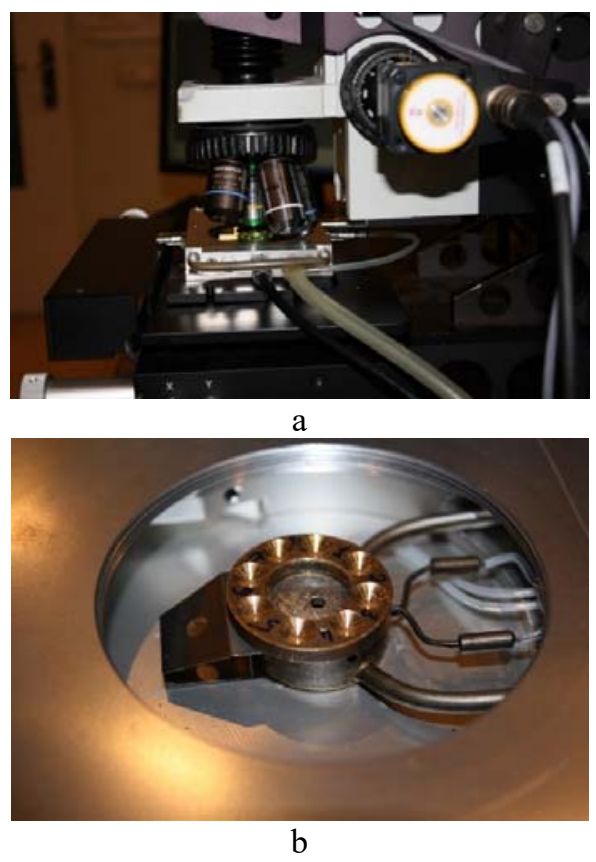

Fig. 3. General view of the spectrometer (a) in the process of excitation and registration of the Raman spectra and chamber (b) for the determination of the samples of HAM to be studied.

To be more accurate, for having the Raman spectra registered Horiba Jobin Yvon T64000 triple spectrometer, being equipped with a confocal optical microscope, is contrived to be used. At the same time for the excitation process of optical spectra the radiation of the $\operatorname{Ar}-\mathrm{Kr}$ laser $\left(\lambda_{\text {las }}=514.5 \mathrm{~nm}\right)$ was considered to be advantageously instrumental. As for the laser beam, it possessed the ability to be focused on a spot with a diameter of 0.7 microns.

\section{Results and discussions}

It must be noted that 2 types of nanoparticles, made of carbon and copper, were available for being used as filler materials. Besides, a study of the structural properties of the initial powders was additionally constituted with the ultimate aim of carrying out analysis of the effect of metal micro- and nanoparticles on the dynamics of phase transitions in a mixture of paraffin with specific nanofiller. Particular attention must be paid to the fact, verified with experimental work, proving the idea that the distribution of metal micro- and nanoparticles in the volume of the main substance mainly depends on the size of the particles.

In composite materials, having fractions of nanoparticles of $0.2 \mathrm{~mm}$ or larger, the particles were noticed to be settled on the bottom of the chamber after paraffin melting. As a result, this issue initiated their alleviation effect on the processes of heat accumulation in accumulators. Therefore, this work implicates the approach of having experimental studies carried out on metal nanoparticles for the sedimentation effect to be avoided.

Besides, the thermophysical properties of pure paraffin and paraffin with fillers study was assumed to be done by optical methods, revealing the dependence of $\mathrm{S}_{\text {lateral }}$ parameter on temperature. This aforesaid parameter characterizes the degree of ordering the structure of the material which was provided for studying experience and its dependence on the temperature. To be more precise, depending on the value of the $S_{\text {lateral }}$ parameter the material under study may be arranged to be in a crystalline state, in the process of melting or, on the contrary, it may be disarranged being in a liquid state. In particular, the obtained dependences provide an invaluable opportunity for the melting temperature of different materials to be determined.

Figure 4 illustrates the dependences of $\mathrm{S}_{\text {lateral }}$ parameter on the temperature for pure paraffin $\mathrm{T} 3$ (curve 1), T3 paraffin with carbon nanoparticles with a size of $0.1 \mathrm{~mm}$ (curve 2) and copper nanoparticles with a size 0.07-0.1 mm (curve 3).

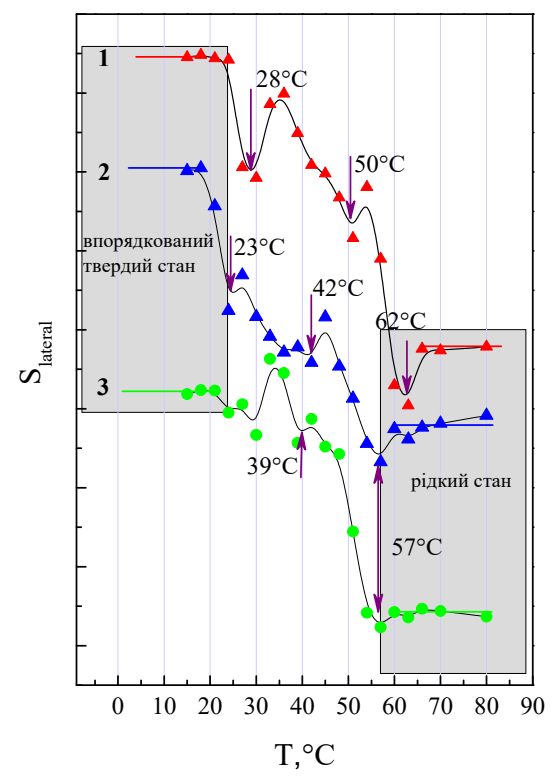

Fig. 4. Comparison of the temperatures of the structural and phase transitions of paraffin T3 (1), a mixture of paraffin $\mathrm{T} 3$ with carbon filler with size 0.1 $\mathrm{mm}(2)$ and copper filler (3) with size 0.07-0.1 mm.

As shown in Figure 4, the melting point for pure paraffin is $58-60{ }^{\circ} \mathrm{C}$, for the composite with carbon nanoparticles item is $57-59{ }^{\circ} \mathrm{C}$ and, accordingly, the composite with copper particles issue is $54-56{ }^{\circ} \mathrm{C}$. As a consequence, it can obviously be summed up that there is a tendency that the presence of metal nanoparticles in paraffin leads to a decrease in the melting point by 
$7-9 \%$. So, the increase in the effective coefficient of thermal conductivity for composite materials is clearly indicated.

Furthermore, to meet the need of getting research upon the melting processes and the dynamics of temperature distribution in HAM with phase transition, experimentally based studies (Figure 1, 2) were performed. They were carried out with more precision and certainty under laboratory conditions having pure paraffin and paraffin with copper filler with a fraction size of $0.07-0.1 \mathrm{~mm}$ (volume ratio $20: 1$ ). So, due to the abovementioned investigations of transfer processes during phase transformations of pure and composite paraffin, definite dependence models of temperature indices of HAM on time were reported to be caught on (Figure 5).

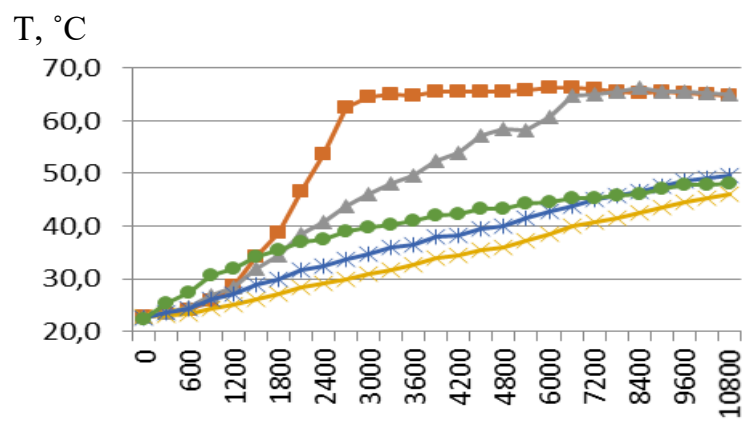

a

Time, s

$\mathbf{T},{ }^{\circ} \mathbf{C}$

Thermocouple number:

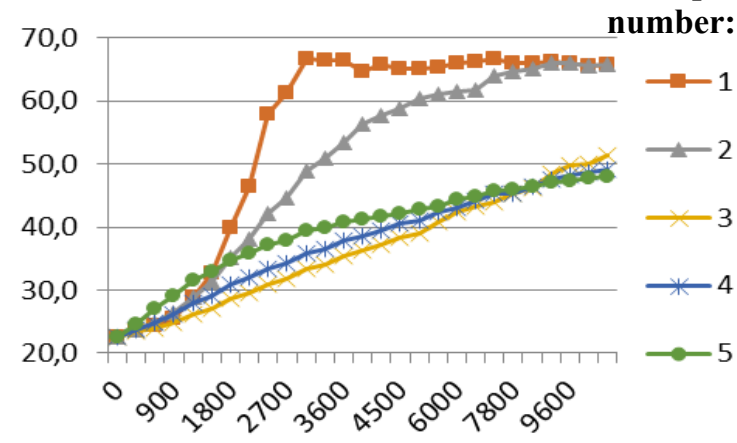

Time, $s$

b

Fig. 5. Dependences of temperatures in the volume of pure paraffin (a) and paraffin with copper micro- and nanoparticles: curves 1-5 correspond to temperature indicators on thermocouples 1-5, which are given in the Table 1.

The data analysis process of the obtained dependences finally discusses the found interpretation about an increase in temperature throughout the volume in the presence of copper fraction compared with pure paraffin, which is averaged to $4-6 \%$. To be exact, this fact attempts to frame the idea of an increase in the thermal conductivity of composite paraffin with copper nanoparticles.

Finally, the result definitely recalls that after careful fact consideration it was established beyond any doubt that such an increase led to an acceleration of the melting of HAM, being located above the heat source by $10-12 \%$ and $20-22 \%$ for it. This state is considered to be at or above the axial line of the heat source. It must be emphasized that for strict observation such kinds of statistics can be achieved using the same reproducible experimental techniques performed on high accuracy level under the same conditions.

In addition, heat accumulation processes in the volume of the HAM case, having the geometric dimensions corresponding to the dimensions of the experimental installation case, has been numerically investigated and verified. The walls of the case were assumed to be insulated and the power and dimensions of the cylindrical heat source were reported to coincide with the power of HES in the constructed set-up.

It must be indicated that the obsolete analysis of numerical simulation relating pure paraffin $\mathrm{T} 3$ and paraffin with copper filler, which has the size of $0.07-0.1 \mathrm{~mm}$, was delivered. The mathematical model of heat and mass transfer processes in the phase transformations of the accumulation material can be approximated by applying the Navier-Stokes equation and the convective heat transfer equation with the use of the package of applications COMSOL Multiphysics3.5a.

Hence, the result of numerical simulation, the temperature distributions in the volume of accumulative material for pure paraffin and paraffin with copper filler can be visually presented in Figure 6. Further observation of analysis of the obtained distributions delivers the idea of having the average temperature indices in the composite HAM as being 6-9 \% higher than those in pure paraffin, which agrees with the data experimentally achieved.

$\mathbf{T},{ }^{\circ} \mathbf{C}$

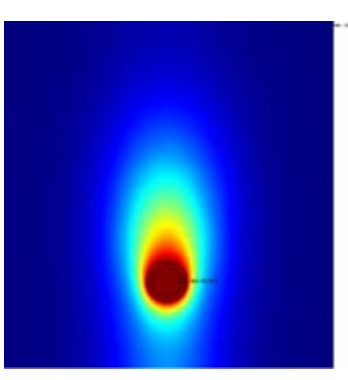

a

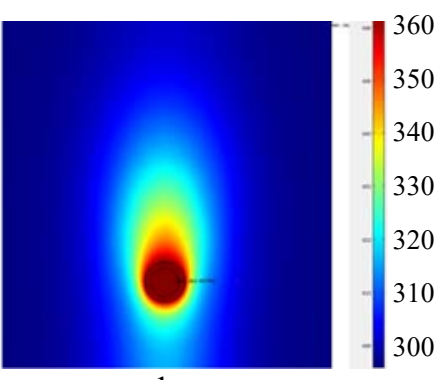

b
Fig. 6. Temperature distributions in volume of HAM: $\mathrm{a}$ - pure paraffin; $\mathrm{b}$ - paraffin with copper nanoparticles.

Thus, numerical and experimental studies provided the opportunity to document and verify the thesis statement that the use of HAMs during phase transformations in the presence of metal nanoadditives in them confirms the validity of being quite beneficial for improving the thermal conductivity of such materials, as it sufficiently increases the melting speed of these materials and, as a result, the efficiency of accumulators with phase transformations.

\section{Conclusions}

In general, numerical and experimental studies reached the mutual agreement on the point that the addition to the 
heat-accumulation materials with phase transition of metal fractions with small micro- and nanoscales makes possible improving the heat-accumulation properties of such materials.

In particular, the following conclusion can be drawn from the obtained results:

1. Temperature dependences of the parameter characterizing the arrangement and disarrangement of pure paraffin and their mixtures with metal conductive fillers are reported to be acquired through optical spectroscopy methods using practice. Moreover, the analysis of these dependencies concedes the ability to have the temperature of phase transitions, both in pure technical paraffin, and in paraffin with metal particles of different chemical composition of sub-millimeter sizes determined.

2. The dynamics of structural-phase transformations for pure paraffin and paraffin with thermal conductive fillers are regarded to be investigated and the differences of these transformations are managed to be established.

3. The data analysis provides additional evidence to thesis defense that the presence of metallic nanoparticles in the phase-transfer material cause heat conductivity increase and even the distribution of the thermal field and a decrease in the temperature of phase transitions in heat-accumulation materials. Besides, the increase in the average temperature throughout the volume of heataccumulation materials with phase transformation in the presence of metallic nanofraction appears to have been found out. It is calculated as being equal to $4-6 \%$ on average. Furthermore, the melting speed of heataccumulation materials with nanoparticles was reported to be increased being placed over the heat source by $20-22 \%$ benefit.

4. Finally, experimentally based researches combined with numerical studies substantiated with confidence to have the feasibility validation of using thermal conductors from metallic micro- and nanoparticles for the process of phase transformations in pure paraffin to be intensified and for the efficiency of heat accumulators to be increased.

\section{Acknowledgments}

Publication is based on the research provided by the grant support of the State Fund for Fundamental Research of Ukraine, the Ministry of Education and Science of Ukraine (project N F64/11-2016).

\section{References}

1. J. Pereira da Cunha, P. Eames, Appl. Energ., 177, pp. 227-238 (2016)

2. L. Liu, D. Su, Y. Tang, G, Fang, A review. Renew. Sust. Energ. Rev., 62, pp. 305-317 (2016)

3. L. Fan, J. Khodadadi, A review. Renew. Sust. Energ. Rev., 15(1), pp. 24-46 (2011)

4. F. Agyenim, N. Hewitt, P. Eames, M. Smythet, Renew. Sust. Energ. Rev, 14(2), pp. 615-628 (2010)
5. Y. Huo, Z. Rao, Int. J. Heat Mass Tran, 86, pp. 197206 (2015)

6. A. Sharma, V.V. Tyagi, C.R. Chen, D. Buddhi, Renew. Sust. Energ. Rev, 13(2), pp. 318-345 (2009)

7. Q. Zhang, Y. Huo, Z. Rao, Science Bulletin, 61(5), pp. 391-400 (2016)

8. A. Trp, Sol. Energy, 79, pp. 648-660 (2005)

9. S. Kalaiselvam, M. Veerappan, A. Arul, S. Iniyan, Int. J. Therm. Sci., 47(7), pp. 858-874 (2008).

10. A. Regin, S. Solanki, J. Saini, Renew. Energ., 31, pp. 2025-2041 (2006)

11. I. Antypov, Bulletin of ARIEA, 1(16), pp. 196-200 (2016)

12. V. Gorobets, I. Antypov, V. Trokhaniak, Yu. Bohdan, MATEC Web of Conferences 2018, 240, 01009

13. S. Jegadheeswaran, D. Pohekar, Renew. Sust. Energ. Rev., 13(9), pp. 2225-2244 (2009)

14. S. Kuboth, A. König-Haagen, D. Brüggemann, Energies, 10, 274 (2017)

15. Nabeel S. Dhaidan, J.M. Khodadadi, Tahseen A. AlHattab, Saad M. Al-Mashat, Int. J. Heat Mass Transfer, 66, pp. 672-683 (2013)

16. S. H. Tasnim, R. Hossain, S. Mahmud, A. Dutta, Int. J. Heat Mass Transfer, 8, pp. 206-210 (2015)

17. C. Kaviarasu, D. Prakash, J. Eng. Sci. Technol. Rev., 9(4), pp. 26-36 (2016)

18. K. Kant, A. Shukla, A. Sharma, P.H. Biwole, Solar Energy, 146 (C), pp. 453-463 (2017)

19. M. Sepehr, S. Majid, M. Mohammad, Part A: Applications, An International Journal of Computation and Methodology, 75(85), pp. 560-577 (2019)

20. M.A. Said, H. Hamdy, Energy Conversion and Management, 171(1), pp. 903-916 (2018)

21. Iu. Nasieka, V. Strelchuk, V. Naseka, Yu. Stubrov, S. Dudnik, V. Gritsina, O. Opalev, K. Koshevoy, V. Strel'nitskij, V. Tkach, M. Boyko, I. Antypov, J. Cryst. Growth, 491, pp. 103-110 (2018) 